\title{
The Electric Quadrupole Moment of $\operatorname{In}^{115}$
}

\author{
R. F. Bacher and D. H. Tomboulian \\ Cornell University, Ithaca, New York \\ (Received August 16, 1937)
}

\begin{abstract}
Measurements of the lines $\lambda 7852\left(5 s 6 p^{1} P \rightarrow 5 s 6 s^{1} S\right)$ are $\lambda 8241\left(5 s 6 p^{1} P \rightarrow 5 s 5 d^{1} D\right)$ of In II show deviations from interval rule. These deviations are satisfactorily accounted for by the presence of a nuclear electric quadrupole moment which from the first of the lines is found to be $Q=0.82 \times 10^{-24} \mathrm{~cm}^{2}$. No trace of lines due to $\operatorname{In}^{113}$ was found.
\end{abstract}

$\mathbf{M}^{\mathrm{E}}$ EASUREMENTS on the hyperfine structure of the lines of indium II by Paschen and Campbell ${ }^{1}$ have shown that the nuclear spin is $4 \frac{1}{2}$ from the use of the interval rule. Several terms of the $5 s n s$ and $5 s 6 p$ configurations lead uniquely to this value for the nuclear spin. Further work by Paschen ${ }^{2}$ has showed that the interval rule is followed more closely by the low ${ }^{3} S$ terms than by the ${ }^{3} P$ and ${ }^{3} D$ terms. The higher ${ }^{3} S$ terms show marked deviations from the interval rule. These deviations and those in the ${ }^{3} P$ and ${ }^{3} D$ terms are due mainly to the presence of nearby levels so that perturbations occur between terms of the same total angular momentum $F$. Paschen has discussed these deviations for higher series members in some detail. In order to ascertain whether there were also deviations from interval rule which were not due to perturbations of this sort but which might arise if the nucleus possessed an electric quadrupole moment, lines involving the $5 s 6 p^{1} P$ and $5 s 5 d^{1} D$ terms were investigated. ${ }^{3}$ The $5 s 6 p^{1} P$ term is sufficiently removed from the ${ }^{3} P$ term of the same configuration so that perturbation of the type mentioned is negligible. This is also true for the $5 s 5 d^{1} D$ term but, due to a different type of perturbation from the $5 p^{2} 1 D$, it is not useful for determining the quadrupole moment. This latter perturbation does not affect the interval rule however, and the observed deviations may be attributed to electric quadrupole moment. ${ }^{3}$

The indium spectrum was excited in a hollowcathode discharge tube operated under a variety of conditions. The cathode was run hot and the

\footnotetext{
1 Paschen and Campbell, Naturwiss. 22, 136 (1934).

2 Paschen, Sitzungsbericten der Preussischen Akademie der Wissenschaften 456 (1934); 431 (1935).

${ }^{3}$ Bacher and Tomboulian, Phys. Rev. 50, 1096 (1936).
}

current in the lamp frequently exceeded one ampere. This did not seem to broaden the lines appreciably. The radiation was studied with a Fabry-Pérot etalon with fixed separators used in conjunction with a Zeiss three prism spectrograph. The interferometer and the spectrograph were kept in a constant temperature room and the interferometer remained in adjustment for days at a time though the exposures were never longer than two hours. For accurate measurements, it was found advisable to use small grain, high contrast plates, though when weaker components were sought the faster plates were used. Most attention was confined to the lines $\lambda 7852$ $5 s 6 p{ }^{1} P \rightarrow 5 s 6 s{ }^{1} S$ and $\lambda 82415 s 6 p^{1} P \rightarrow 5 s 5 d^{1} D$. For the first of these lines a rather large number of photographs were taken and the separations reduced in order to get an idea of the deviations which might be expected in measurements on a simple well resolved line. For this purpose 3, 5 and $8 \mathrm{~mm}$ separators were used. The separators completely change the relative spacings of the components as can be seen in Fig. 1. For this line about ten orders of the interference pattern were measured on each plate.

Table I shows the results obtained for different plate separations for the three component line $\lambda 7852$. The separations show maximum deviations from the mean of $2 \times 10^{-4} \mathrm{~cm}^{-1}$ and $3 \times 10^{-4}$ $\mathrm{cm}^{-1}$ for the larger and smaller intervals respectively. There does not seem to be any dependence of the measured interval on the interferometer separator which was used. Such a dependence might have been expected had certain systematic errors which were due to the relative spacing of the observed components, been present in the measurements. It seems that the measurements of a completely resolved and well separated pattern are really very accurate. The measure- 


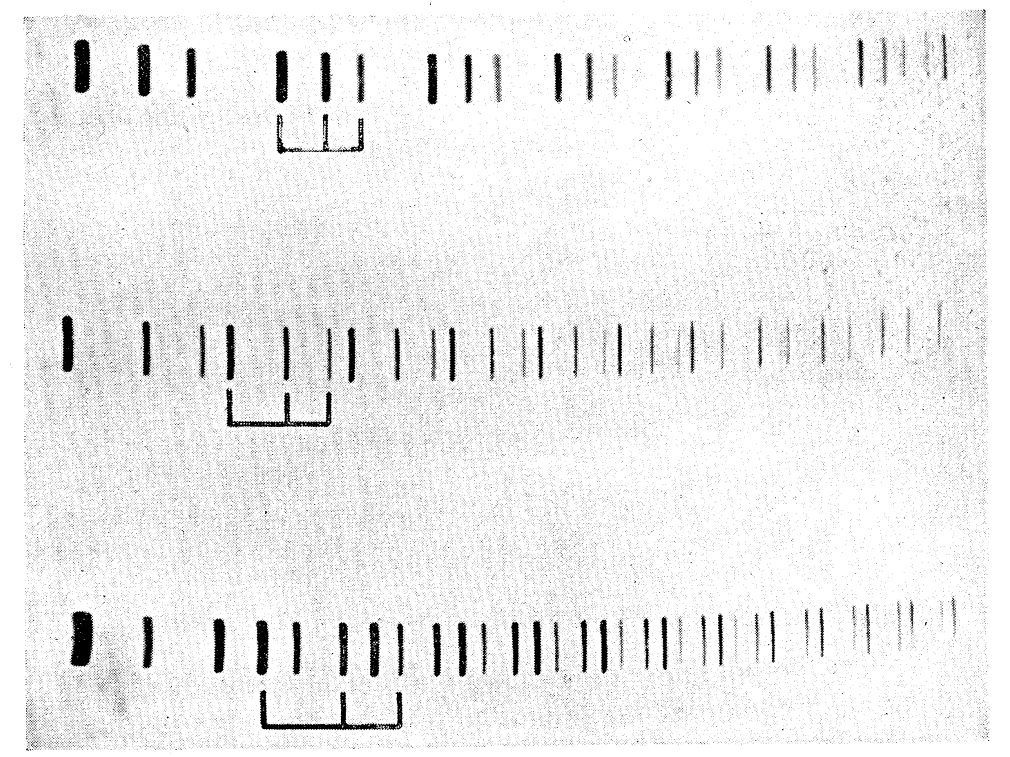

FIG. 1. Interferometer patterns of $\lambda 7852\left(5 s 6 p{ }^{1} P \rightarrow 5 s 6 s{ }^{1} S\right)$ taken with 8 (bottom), 5 (middle) and $3 \mathrm{~mm}$ separators.

ments of $\lambda 7852$ give the hyperfine separations of the ${ }^{1} P$ term directly as seen from Fig. 2 . The separations of the ${ }^{1} P$ state show definite deviations from the interval rule. Using the same over-all separation, the central level would necessarily be so placed that the two hfs. separations would be 357.7 and $437.1 \times 10^{-3} \mathrm{~cm}^{-1}$ (Fig. 2) if the interval rule holds. The values differ from the observed separations by $5.2 \times 10^{-3}$ $\mathrm{cm}^{-1}$ which is respectively 17 and 26 times the maximum deviation found in these measurements.

The interval rule follows directly from the simple form of interaction between the nuclear magnetic moment and the electrons. A deviation from the interval rule does not mean that this form of the interaction is insufficient or incorrect. Such deviations are frequently found where two states of different angular momentum $J$ approach each other. If the distance of such states approaches the hyperfine separations, then large deviations are expected. The deviations are appreciable for separations of the states of different $J$ of $100 \mathrm{~cm}^{-1}$ or more. In the present case of $5 s 6 p^{1} P$ the nearest states are those of the $5 s 6 p^{3} P$. All of these should perturb the ${ }^{1} P$ slightly but the effect of the ${ }^{3} P_{0}$ would be greatest since it would affect only the central hyperfine level of ${ }^{1} P_{1}$. This perturbing effect would be less than $10^{-4} \mathrm{~cm}^{-1}$ and is therefore neglected here. No other perturbations are expected and it appears that the deviations from the interval rule are due to the inadequacy of the interaction term. They can be accounted for by assumption of an electric quadrupole moment for the nucleus as found in other cases.

That this explanation is plausible will be seen from the measurements on the nine component line $\lambda 8241 \quad\left(5 s 6 p^{1} P_{1} \rightarrow 5 s 5 d^{1} D_{2}\right)$. This line is considerably more complex and the measurements of the intervals less accurate. Fig. 3 shows the level scheme and pattern of the line and

TABLE I. Separations of the $5 s 6 p{ }^{1} P_{1}$ state of In II. The separations of the ${ }^{1} P_{1}$ state were measured from $\lambda 7852$ $\left(5 s 6 p^{1} P \rightarrow 5 s \sigma s^{1} S\right)$.

\begin{tabular}{|c|c|c|c|c|}
\hline $\begin{array}{c}\text { SEPARATOR } \\
\text { MM }\end{array}$ & $\begin{array}{l}\text { INTERVAL } \\
11 / 2-9 / 2 \\
10^{-3} \mathrm{CM}^{-1}\end{array}$ & $\begin{array}{c}\text { DEV. FROM } \\
\text { MEAN } \\
10^{-3} \mathrm{CM}^{-1}\end{array}$ & $\begin{array}{c}\text { INTERVAL } \\
9 / 2-7 / 2 \\
10^{-3} \mathrm{CM}^{-1}\end{array}$ & $\begin{array}{c}\text { DEV. FROM } \\
\text { MEAN } \\
10^{-3} \mathrm{CM}^{-1}\end{array}$ \\
\hline 3.1226 & 432.0 & +0.1 & 363.2 & +0.3 \\
\hline 3.1223 & 431.9 & 0.0 & 362.8 & -0.1 \\
\hline & 432.0 & +0.1 & 362.8 & -0.1 \\
\hline “" & 431.7 & -0.2 & 362.9 & 0.0 \\
\hline 5.0136 & 432.1 & +0.2 & 362.7 & -0.2 \\
\hline & 431.7 & -0.2 & 362.6 & -0.3 \\
\hline “ & 431.7 & -0.2 & 363.0 & +0.1 \\
\hline 8.0083 & 431.9 & 0.0 & 362.8 & -0.1 \\
\hline 0.000 & 432.1 & +0.2 & 363.2 & +0.3 \\
\hline “ & 432.0 & +0.1 & 362.9 & 0.0 \\
\hline Mean & 431.9 & & 362.9 & \\
\hline
\end{tabular}




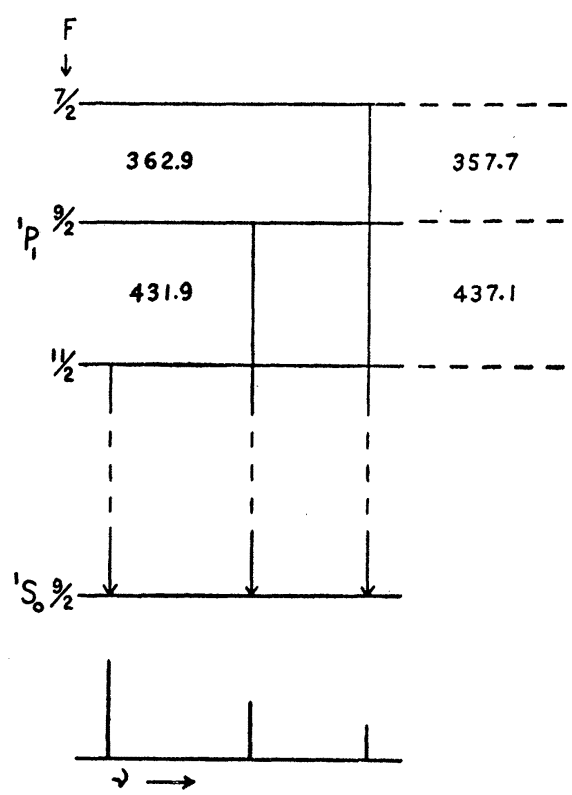

FIG. 2. Level scheme and line structure observed for $\lambda 7852$.

Table II gives the measured separations of the ${ }^{1} D_{2}$ level. Component 3 was too weak for accurate measurements and components 8 and 9 were resolved only in one or two cases and their measured separations thus unreliable. Fig. 4 shows the separations of the ${ }^{1} D_{2}$ level observed, and also by using the over-all separation to determine the interval factor and assuming the interval rule to be correct. The center of gravity found from the two unresolved lines was used. These deviations are not due to perturbations for there are no perturbing levels nearer than $3700 \mathrm{~cm}^{-1}$. The $5 s 5 d^{1} D$ is, however, perturbed by the $5 p^{2}{ }^{1} D$ but the interval rule is not affected thereby. The deviations must be due to a different form for the interaction energy presumably from the presence of an electric quadrupole moment. Such a moment gives rise to a characteristic change in the hyperfine separations. The separation of any two hyperfine levels $F$ and $F^{\prime}$ can be written ${ }^{4}$

$$
\begin{aligned}
\Delta\left(F-F^{\prime}\right)=\frac{1}{2} A & \left(K-K^{\prime}\right) \\
& +\frac{3}{8} B\left\{K(K+1)-K^{\prime}\left(K^{\prime}+1\right)\right\},
\end{aligned}
$$

where $K=F(F+1)-I(I+1)-J(J+1)$

${ }^{4}$ See H. B. G. Casimir "On the Interaction between Atomic Nuclei and Electrons." Prize Essay published by "Tyler's Tweede Genootschap."

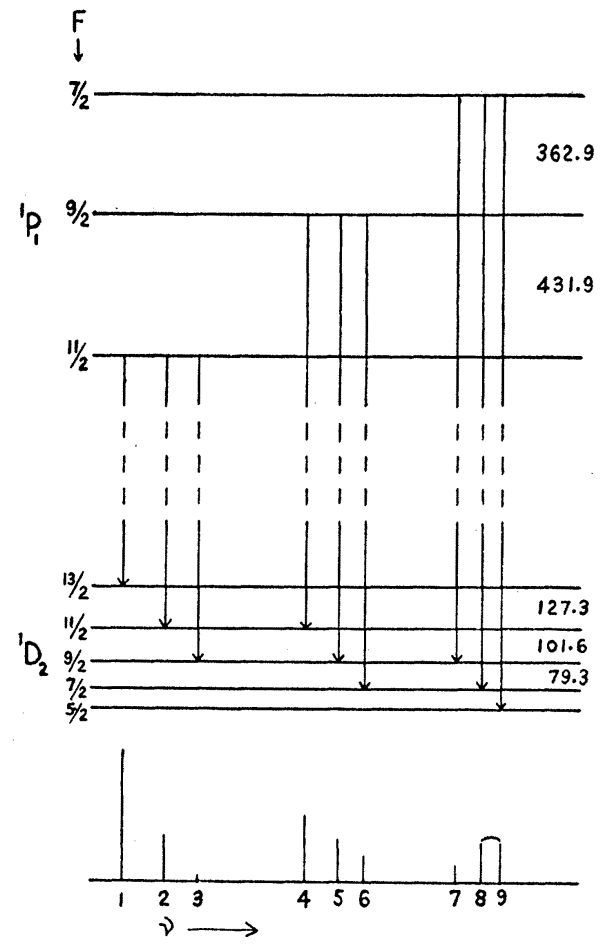

FIG. 3. Level scheme and line structure observed for $\lambda 8241$.

and $A$ and $B$ are constants, the first associated with the separation due to the nuclear magnetic moment and the second arising from the contribution of the electric quadrupole moment. The observed values of the two largest separations of $5 s 5 d^{1} D_{2}$ have been used to determine $A=18.47$ and $B=0.0615 \times 10^{-3} \mathrm{~cm}^{-1}$ and the remaining separations and over-all separation determined from them. In Fig. 4 the levels on the right show the separations so determined. The agreement indicates that the assumption of an electric quadrupole moment is justified. The determination of the moment itself from the above value of $B$ cannot be carried out with any

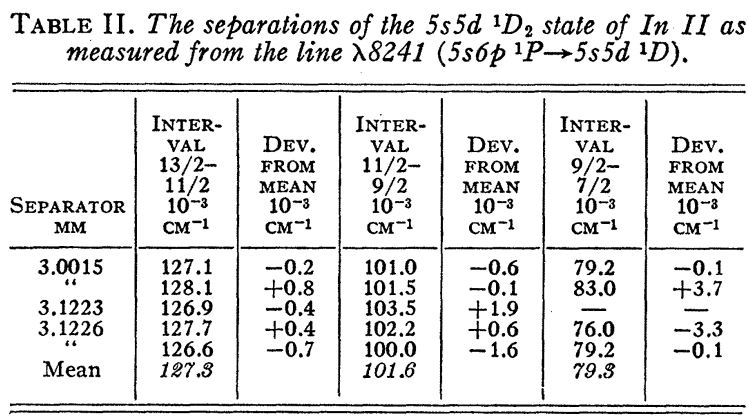


degree of accuracy for this level due to the perturbation by $5 p^{2} 1 D$ which makes the character of the level uncertain.

The value of the nuclear electric quadrupole moment can be found from a consideration of the $5 s 5 p^{1} P_{1}$ level. From the observed separations using (1), $A=-79.37 \times 10^{-3} \mathrm{~cm}^{-1}$ and $B=0.141$ $\times 10^{-3} \mathrm{~cm}^{-1}$. In the case of only three levels there is no check on the value of $B$. The value of the quadrupole moment $t^{4}$ in terms of $B$ is found to be

$$
Q=\frac{-B J(2 J-1) I(2 I-1)}{7.9\left\langle\left(\left(3 \cos ^{2} \theta-1\right) / r^{3}\right)_{J J}\right\rangle_{\mathrm{Av}}}
$$

where $\left\langle\left(\left(3 \cos ^{2} \theta-1\right) / r^{3}\right)_{J J}\right\rangle_{\mathrm{Av}}$ depends upon the electron configuration, the coupling and upon relativistic corrections and $B$ is given in units $10^{-3} \mathrm{~cm}^{-1}$. For $s p^{1} P_{1}$,

$$
\begin{aligned}
& \left\langle\left(\frac{3 \cos ^{2} \theta-1}{r^{3}}\right)_{J J}\right\rangle_{\mathrm{Av}}= \\
& -\left\{c_{1}^{2} \frac{(2 l-1)(l+2) 2 l}{(l+1)(2 l+1)(2 l+3)}\left\langle\frac{1}{r^{3}}\right\rangle_{\mathrm{Av}}^{\prime, '}\right. \\
& \left.\quad-\frac{12 c_{1} c_{2}[l /(l+1)]^{\frac{1}{2}}}{(2 l+1)(2 l+3)}\left\langle\frac{1}{r^{3}}\right\rangle_{\mathrm{Av}}^{\prime, ' \prime}\right\} .
\end{aligned}
$$

The factors $c_{1}$ and $c_{2}$ depend on the coupling

$$
c_{1}=\cos \left(\theta-\theta_{0}\right) ; \quad c_{2}=-\sin \left(\theta_{0}-\theta\right),
$$

where

$$
\theta_{0}=\arctan [l /(l+1)]^{\frac{1}{2}}
$$

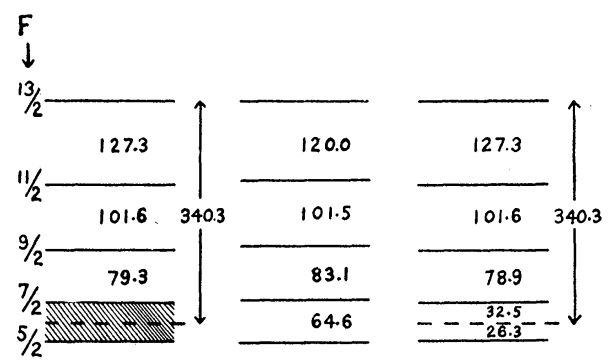

FIG. 4. Measured lintervals of $5 s 5 d^{1} D_{2}$ are shown on the left. The center sketch shows the separations obtained assuming the interval rule and with the separation constant determined from the over-all separation. On the right are the calculated levels found by using the two larger level separations for the determination of $A$ and $B$. and $\sin ^{2} \theta=\Delta / d, \Delta$ being the deviation of the ${ }^{3} P_{1}$ term from its position if the interval rule held exactly and $d$ the ${ }^{3} P_{1}-{ }^{1} P_{1}$ separation. $\left\langle 1 / r^{3}\right\rangle_{\mathrm{Av}}{ }^{\prime}{ }^{\prime}$, and $\left\langle 1 / r^{3}\right\rangle_{A v}{ }^{\prime}, "$ are equal to certain relativistic correction terms ${ }^{4}$ times $\left\langle 1 / r^{3}\right\rangle_{\mathrm{Av}}$.

For indium $5 s 6 p, c_{1}=0.915$ and $c_{2}=-0.404$

$$
\left\langle\left(\left(3 \cos ^{2} \theta-1\right) / r^{3}\right)_{J J}\right\rangle_{\mathrm{Av}}=-0.425\left\langle 1 / r^{3}\right\rangle_{\mathrm{Av}} .
$$

The value of $\left\langle 1 / r^{3}\right\rangle_{\mathrm{Av}}$ can be obtained in terms of the doublet separation, or here, in order to include the screening of the $5 s$ electron, from the $5 s 6 p^{3} P$ separation. $\left\langle 1 / r^{3}\right\rangle_{\mathrm{Av}}$ can be found to be

$$
\left\langle 1 / r^{3}\right\rangle_{\mathrm{Av}}=\delta /\left\{H Z_{i}\left(l+\frac{1}{2}\right) R \alpha^{2}\right\} .
$$

Here $\delta$ is the separation, $H$ a relativistic correction factor ${ }^{4} 1.06, Z_{i}$ is taken as $(Z-4)$ and $\left\langle 1 / r^{3}\right\rangle_{\mathrm{AV}}=1.84$ which gives

$$
Q=0.82 \times 10^{-24} \mathrm{~cm}^{2} \text {. }
$$

This value of $Q$ is essentially the same as the value $R^{2}=1.0 \times 10^{-24} \mathrm{~cm}^{2}$ reported previously, ${ }^{3}$ since $Q=2 I R^{2} /(2 I+3)$.

$Q=0.82 \times 10^{-24} \mathrm{~cm}^{2}$ is also in agreement with the value $Q=0.8 \pm 0.2$ recently reported by Schüler and Schmidt ${ }^{5}$ from a study of the $5 p^{2} P_{3 / 2}$ term of In I.

A considerable search was made to find evidence of $\operatorname{In}^{113}$ expected $^{6}$ to be present to the extent of about 4.5 percent. Though long exposures of several lines were made which clearly showed components whose intensity represented only 2 percent of the total intensity of the line, no trace of components attributable to $\mathrm{In}^{113}$ was found. This indicates that the components of $\mathrm{In}^{113}$ are probably concealed under those of $\operatorname{In}^{115}$ which means that the angular momenta and magnetic moments are probably the same for the two isotopes; $I=9 / 2, \mu=5.7$ nuclear magnetons. Such a value for the nuclear spin of $\operatorname{In}^{113}$ is quite adequate $^{7}$ to explain the stability of this isotope and $\mathrm{Cd}^{113}$, since the change from one to the other would be highly forbidden with $\Delta I=4$.

\footnotetext{
${ }^{5}$ Schüler and Schmidt, Zeits. f. Physik 104, 468 (1937).

6 Aston, Proc. Roy. Soc. 149, 396 (1935); Sampson and Bleakney, Phys. Rev. 50, 456 (1936).

${ }^{7}$ Bethe and Bacher, Rev. Mod. Phys. 8, 200 (1936).
} 


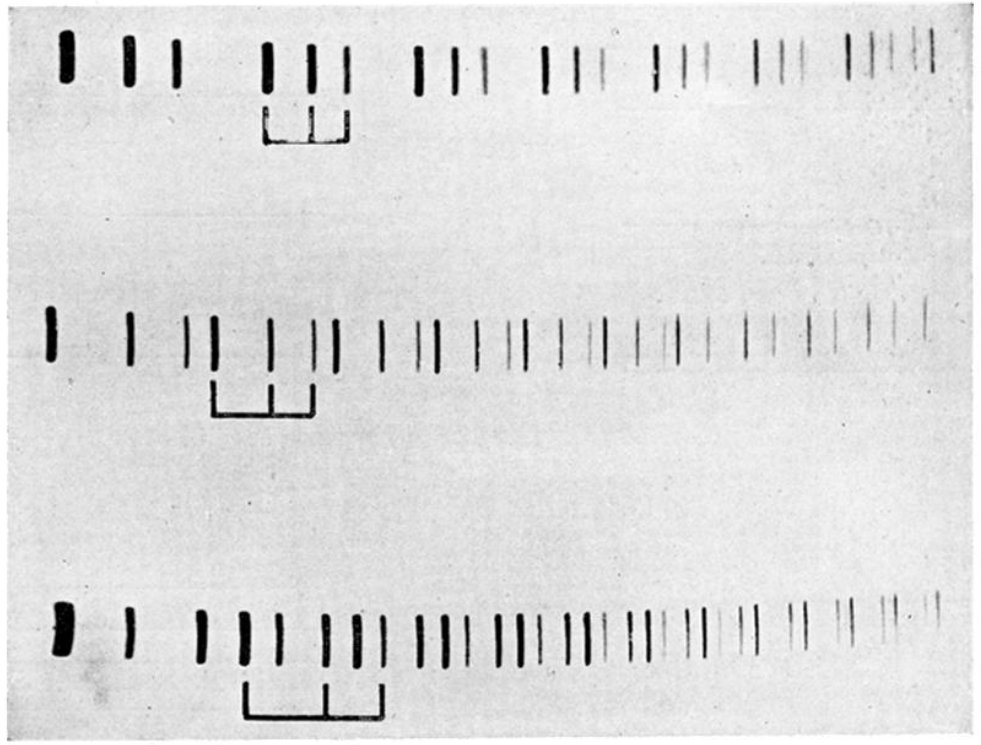

FIG. 1. Interferometer patterns of $\lambda 7852\left(5 s 6 p^{1} P \rightarrow 5 s 6 s^{1} S\right)$ taken with 8 (bottom), 5 (middle) and $3 \mathrm{~mm}$ separators. 